\title{
Rescue of virulent class I Newcastle disease virus variant 9a5b-D5C1
}

\author{
Yang Yu ${ }^{1,2}$, Xusheng Qiư ${ }^{2}$ Dan Xư ${ }^{2}$, Yuan Zhan ${ }^{2}$, Chunchun Meng ${ }^{2}$, Nana Wei ${ }^{2}$, Hongjun Chen², Lei Tan², \\ Shengqing $Y u^{2}$, Xiufan Liu', Aijian Qin ${ }^{1}$ and Chan Ding ${ }^{2,3^{*}}$
}

\begin{abstract}
Background: The virulent class I Newcastle disease virus (NDV) variant 9a5b was generated from a nonvirulent NDV isolate Goose/Alaska/415/91 via nine consecutive passages in the chicken air sac, followed by five passages in the chick brain. The evolutionary mechanism of virulence in the class I NDV isolate is not fully understood. To elucidate this evolutionary mechanism, a reverse genetics manipulation specific for class I NDV is indispensable.

Results: A full-length cDNA clone of 9a5b and the helper plasmids $\mathrm{pCl}-\mathrm{NP}, \mathrm{pCl}-\mathrm{P}$, and $\mathrm{pCl}-\mathrm{L}$ were constructed from segments of cDNA. After these plasmids were co-transfected into BSR T7/5 cells, infectious viral particles were obtained. The rescued viruses were genetically and biologically identical to the parental strain and showed similar pathogenicity in chickens.

Conclusion: A stable recovery method for class I NDV was established. Reverse genetics of the class I NDV variant $9 \mathrm{a} 5 \mathrm{~b}$ allowed for the generation of genetically altered and virulent NDV, and can be used as a foundation for research on the evolution of virulence in class I NDV isolates.
\end{abstract}

Keywords: Newcastle disease virus, Reverse genetics, Minigenome, Helper plasmids

\section{Introduction}

Newcastle disease (ND) is one of the most serious and lethal avian diseases, resulting in heavy losses within the poultry industry worldwide [1-3]. Its causative agent, virulent Newcastle disease virus (NDV), is classified as the Avulavirus genus within the paramyxovirinae subfamily of the paramyxoviridae $[4,5]$. The genome of the virus is composed of a single-stranded, negative-sense RNA approximately $15 \mathrm{~kb}$ in length that encodes six structural viral proteins: nucleoprotein (NP), phosphoprotein $(\mathrm{P})$, matrix protein $(\mathrm{M})$, fusion protein $(\mathrm{F})$, hemagglutinin-neuraminidase $(\mathrm{HN})$ and the large protein (L) [6]. In addition, two non-structural proteins, $\mathrm{V}$ and $\mathrm{W}$, are also encoded by the $\mathrm{P}$ gene of NDV via the RNA editing mechanism $[7,8]$.

NDV isolates were classified into two major categories class I and class II. Class II NDV includes nine well-

\footnotetext{
* Correspondence: shoveldeen@shvri.ac.cn

${ }^{2}$ Shanghai Veterinary Research Institute, Chinese Academy of Agricultural Sciences, Shanghai 200241, People's Republic of China

${ }^{3}$ China National Engineering Technology Research Centre for Poultry, 2949

Zhennan Road, Shanghai 200331, People's Republic of China

Full list of author information is available at the end of the article
}

established genotypes labeled by Roman numerals. Class I NDV was identified approximately 6 years ago and has since proved to be wide-spread in avian species $[9,10]$. Class II genotypes have been well researched, but the significance of class I isolates is not well understood [11].

Class I NDV has several different poultry hosts, most of which do not experience any observable symptoms of viral infection [12-14]. Although class I virus isolates are not usually virulent, evolution of virulence in the class I strains has been reported. Yu et al passaged the nonpathogenic class I NDV isolate of Goose/Alaska/415/91 nine times through the specific pathogen free (SPF) chicken air sac and, subsequently, five times through the chicken brain [15]. Virulence gradually evolved in the viral isolate; the final generation of the virus was designated $9 \mathrm{a} 5 \mathrm{~b}$ and it displayed virulence equivalent to that of the typical velogenic NDV strain, Herts/33. Few differences in nucleotides were found between the genomes of these viruses. The $\mathrm{F}$ motif had mutated from that in ERQER/L (as in the parent strain, Goose/Alaska/415/91) to resemble the typical virulent type, KRQKR/F; the $\mathrm{HN}$ length was changed from 616 aa to 572 aa; however, no detectable change in the virulence was observed in 
Goose/Alaska/415/91 when it was passaged more than 15 times through embryonated eggs [15,16]. This research has raised several issues that need to be addressed including the relevance of the cellular materials in the chicken air sac compared to that of eggs in the development of pathogenic NDV, and the potential causal or functional relationship between the mutations in $\mathrm{HN}$ and $\mathrm{F}$. In our research, we wanted to determine the mechanisms involved in the process of NDV virulence evolution. Since there is little information available, a reverse genetics manipulation for NDV strain 9a5b-D5C1 was constructed as a foundation to assess NDV virulence evolution.

\section{Results}

\section{Construction of a full-length cDNA clone from the 9a5b-D5C1 genome}

To avoid interference from quasispecies, the original 9a5b strain was plaque-purified by five passages through DF1 cells. During the plaque purification, the biggest plaque from each generation of passage was collected and used for the subsequent passage. The resulting clone 9a5b-D5C1 (designate as D5C1 thereafter) was utilized for the subsequent recovery. Fragments of cDNA clones spanning the entire viral genome were transcribed from viral RNA with 6-nt random primers and then amplified by polymerase chain reaction (PCR) for sequencing. To ensure precision, the genome of $\mathrm{D} 5 \mathrm{C} 1$ was sequenced at least five times. The full length genomic sequence of D5C1 was compiled and submitted to the GenBank (accession number: JQ713944). D5C1 and the original 9a5b genomic sequence (accession number: AB524406.1) had greater than $99.99 \%$ nucleotide identity. Only six nucleotides were found to differ from the consensus reported in Japan: $3628 \mathrm{C}(\mathrm{T}), 7406 \mathrm{~A}(\mathrm{~T}), 9511 \mathrm{C}(\mathrm{T})$, $9513 \mathrm{C}(\mathrm{T}), 9514 \mathrm{C}(\mathrm{T})$, and $9988 \mathrm{G}(\mathrm{A})$ (consensus sequence in parentheses).

Based on verified sequences of D5C1, a NDV fulllength cDNA clone was assembled from seven segments of cDNA and subcloned into vector pTVT7R(0.0) (Figure 1A). In the resulting plasmid, TVT-D5C1, the fulllength NDV cDNA was flanked with the T7 RNA promoter and the hepatitis delta virus (HDV) ribozyme motif.

\section{Verification of helper plasmids}

The co-expression of viral nucleoprotein (NP), phosphoprotein $(\mathrm{P})$ and large protein $(\mathrm{L})$ is essential for recovery of paramyxoviruses. By using the full-length cDNA clone as the template, the complete coding region of the NP, $\mathrm{P}$ and $\mathrm{L}$ genes was obtained using PCR. The NP and $\mathrm{P}$ gene were amplified by high-fidelity RT-PCR and ligated into the $\mathrm{PCI}$ neo vector (Promega, Madison, WI, USA) to obtain the plasmids pCI-NP and pCI-P. Three overlapping PCR products L1, L2 and L3, were amplified and cloned sequentially into the $\mathrm{pCI}$ neo vector to obtain pCI-L. The sequence consistency of the three helper plasmids was verified by sequencing at Sangon Biotechnology (Shanghai, China).

To determine the usefulness of helper plasmids, a minigenome plasmid was prepared as well. In this plasmid, the green fluorescence protein (GFP) gene was flanked by the leader (Le), 5 ' untranslated region (UTR) of NP and 3' UTR of L, the trailer (Tr) (Figure 1B). The Le and $\mathrm{Tr}$ were involved in assembly of mini-genomic RNA by NP protein. 5' UTR of NP contains a transcription start component for viral RNA polymerase, while $3^{\prime}$ UTR of L contains a transcription termination component. The minigenome fragment was inserted into vector pTVT7R(0.0) to obtain plasmid TVT-TGL, which was verified by sequencing.

To examine the efficiency of the helper plasmids pCINP, pCI-P and pCI-L, BSR-T7/5 cells were co-transfected with pCI-NP, pCI-P, pCI-L and pTVT-TGL. In BSR-T7/5 cells, the T7 RNA polymerase will transcribe singlestrand (-) RNA from pTVT-TGL. If the helper plasmids were functional, those plasmid-coded viral proteins will assemble into viral RNA-dependent RNA polymerase (vRdRp) to transcribe single-strand (+) RNA as well as mRNA of GFP from (-) RNA. At 48 h post-transfection, green fluorescence was observed in cells which were transfected with the minigenome pTVT-TGL and helper plasmids. In contrast, there was no visible green fluorescence in those cells in which only TVT-TGL was transfected (Figure 2). This result suggested that the helper plasmids can be successfully used for recovery.

\section{Recovery of infectious recombinant NDV from cDNA}

The full-length cDNA clone, pCI-NP, pCI-P and pCI-L were co-transfected into BSR $\mathrm{T} 7 / 5$, in which the intracellularly-expressed T7 RNA polymerase generated the first strand of viral genomic RNA. Then, vRdRp assembled from NP, P and L was expressed in cells, and the vRdRp participated in the duplication of more viral genomic RNA strands. Two parallel experiments were performed as the controls: the minigenome plasmid TVT-TGL was substituted for the cDNA clone, and pCI-L was removed from the superfection mixture.

After $24 \mathrm{~h}$, the cells transfected with the minigenome started to glow under UV light, suggesting that transfection had been successful. All three kinds of cells were serially passaged (up to three times) in SPF embryonic eggs. The hemagglutination (HA) titers of rescued NDV in the three passages were $2^{7}, 2^{8}$, and $2^{7}$ respectively. As expected, both parallel control experiments displayed no hemagglutination, no matter how many passages were performed.

In order to identify the viruses, rescued viral RNA was extracted from fresh allantoic fluid, and the full-length genome was sequenced as described above. It was found that the genomic sequence of the descendant virus was 


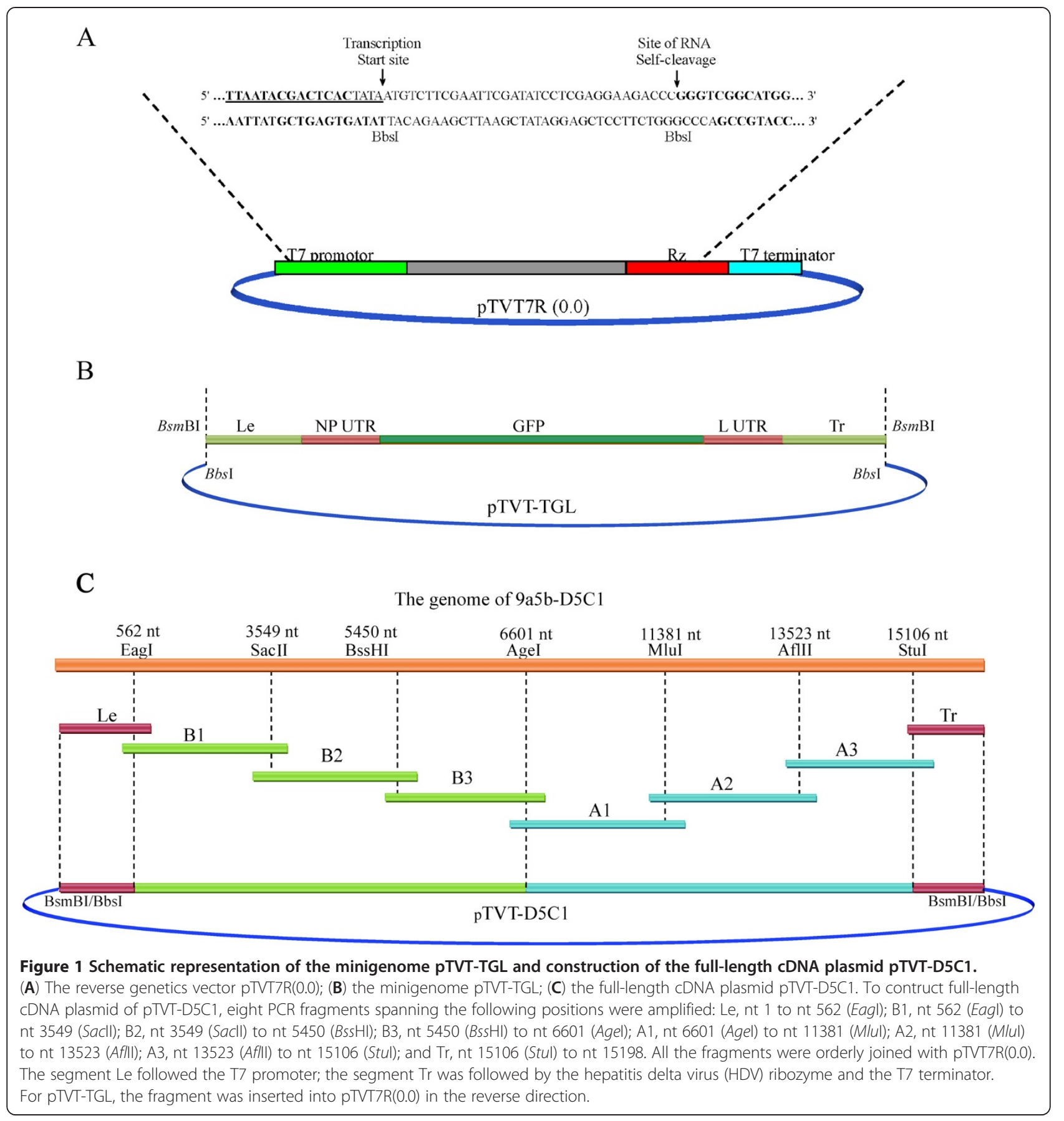

identical to that of the parental virus, with only one exception: $\mathrm{C}$ at nt 14,227 instead of the original T. This unexpected mutation was located in the open reading frame of the $\mathrm{L}$ protein sequence and introduced no amino acid change; the mutation could be considered a genetic marker of rescued virus particles.

\section{Biological characterization of the generated virus}

Three standard pathogenicity tests of mean death time (MDT), intracerebral pathogenicity index (ICPI) and the intravenous pathogenicity index (IVPI), were performed to determine the differences in virulence between rescued $\mathrm{D} 5 \mathrm{C} 1$ and the original virus. The MDT value of the rescued virus was 54 , slightly higher than that of its parental strain. In the ICPI test, all the ten chicks died, and they had an ICPI value of 1.89 . The rescued D5C1 and the original virus also displayed similar values of 2.7 and 2.8, respectively, in the IVPI test. Chickens in both challenge groups were sick $36 \mathrm{~h}$ post-challenge, and all were dead $72 \mathrm{~h}$ post-challenge. Taken together, these 


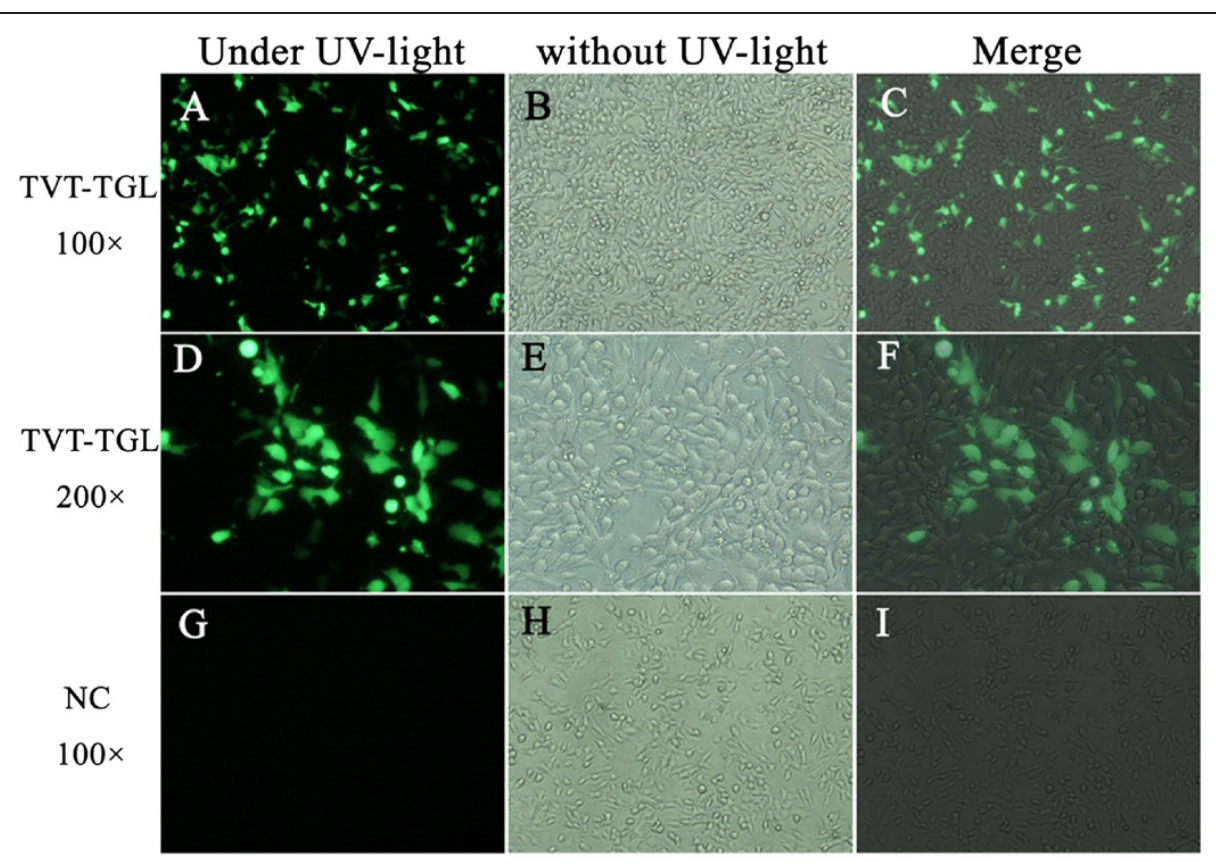

Figure 2 Verification of the helper plasmids pCl-NP, pCl-P and pCI-L in BSR-T7/5 cells co-transfected with minigenome pTVT-TGL. Green fluorescence was observed under fluorescence microscopy in minigenome-transfected cells 48 h post-transfection. Panel "TVT-TGL 100X": Cells transfected with pCI-NP, pCI-P, pCI-L and pTVT-TGL (100x); Panel "TVT-TGL 200x": Cells transfected with pCI-NP, pCI-P, pCI-L and pTVT-D5C1 (200x); Panel "NC 100x": Cells transfected with pTVT-TGL (100x) only. UV-light: ultraviolet.

data show that rescued NDV has a similar pathogenicity as its parental virus.

To compare the propagation capability of these viruses, growth curves were constructed in the DF1 cells for both the parent and the descendent virus. As shown in Figure 3, a similar $\mathrm{TCID}_{50}$ was found at all the time points. This result revealed that the kinetics and rate of replication of rescued NDV are comparable to those of its strain of origin.

\section{Discussion}

Recently, several recombinant Newcastle disease viruses (rNDV) have been generated by genetic techniques [1723]. This manipulation has facilitated the study of the molecular mechanisms behind the virulence, pathogenicity and evolution of NDV. To date, all NDV strains isolated worldwide have been classified into two major categories: class I and class II [10,24-26]. Class II contains the well-researched NDV strain genotypes, while class I viruses were only identified in 2006 and have been studied less intensely [10]. Globally, class I NDVs are commonly isolated from domestic poultry and wild bird species [27-29]. Class I NDV is the dominant class, especially in developed countries where NDV is not endemic in poultry. It is thought that class I NDV interferes with the NDV vaccine [24]. Little is known about the evolution of class I NDV, as most NDV research has been performed on class II viruses.
Class I viruses are generally nonvirulent, except for two known virulent class I viruses [24,30,31]. There is, however, a concern that class I NDVs have the potential to evolve virulence. In 2002, Yu et al reported a virulent class I NDV variant that originated from a nonvirulent

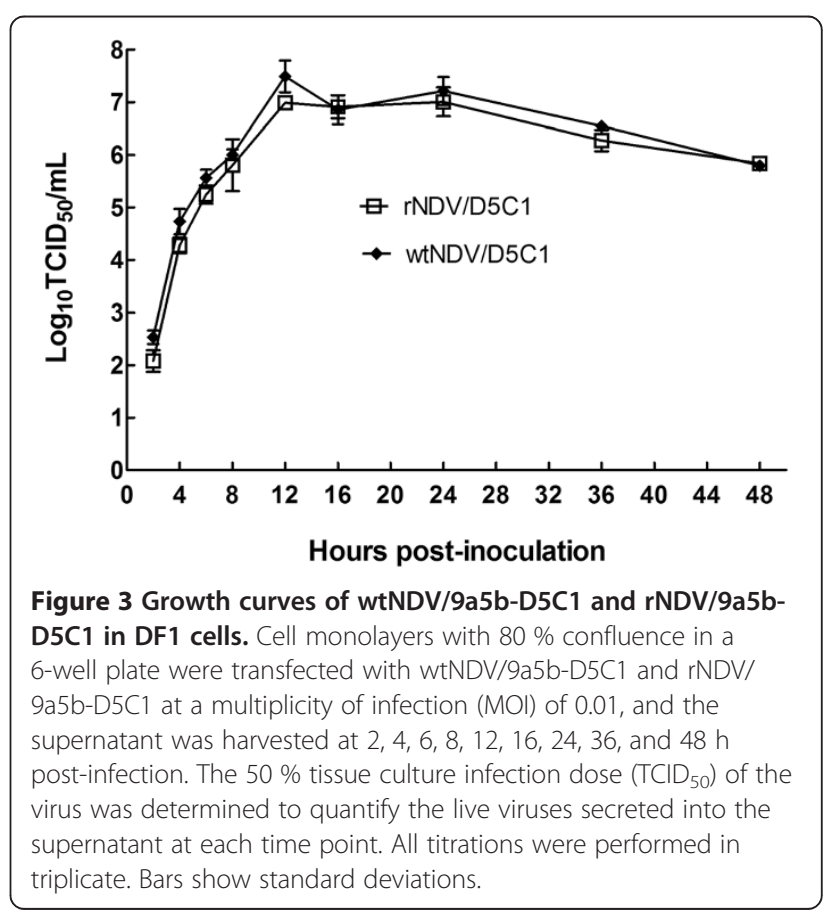


isolate Goose/Alaska/415/91 after nine consecutive passages by air-sac inoculation and five passages through chicken brain [15]. Sequence analysis showed that the fusion protein cleavage site was mutated from KRQKR/F of Goose/Alaska/415/91 to ERQER/L, a typical motif of virulent NDV, in 9a5b [32].

To determine the molecular mechanism behind class I virus evolution, reverse genetics techniques specific for class I NDV are useful; however, all the previous rescued NDVs belongs to class II, and prior to our research, there was no recovery-generated recombinant class I NDV.

In this study, the first virulent recombinant class I NDV variant was generated from the full-length cNDV clone. First, the entire genomic viral cDNA was ligated into the vector pTVT7R(0.0). However, the plasmids containing viral cDNA greater than $12 \mathrm{~kb}$ in length propagated inefficiently in host bacteria cells. Different competent cells, media and plasmid extraction methods did not increase the yield of those plasmids, which maintained a concentration of $50-100 \mathrm{ng} / \mu \mathrm{l}\left(\mathrm{OD}_{260 / 280}\right.$ ranging from 1.8 - 1.9). These results suggested that the genomic cDNA from the class I NDV strain was unstable in the bacterial carrier.

In order to obtain more favorable results, the recovery procedure was modified. All of the plasmids containing a long viral cDNA fragment were identified using a set of specific primer pairs for every $1 \mathrm{~kb}$ of the full-length genome (due to the large number of primers, the data are not shown). Only the positive plasmids were re-transformed into competent cells and extracted with QIAGEN Plasmid Midi Kits for the following experiments.

In spite of the low yield of the full-length cDNA clone, the reverse genetics transfection system used in this study proved to be efficient. The full-length plasmid (50 $\mathrm{ng} / \mu \mathrm{l}$ ) was utilized in the rescue manipulation, and rescued viruses were detected in all three trials containing $20 \mu \mathrm{l}, 25 \mu \mathrm{l}$ or $30 \mu \mathrm{l}$ of full-length plasmid respectively, suggesting that the plasmid was sufficient to rescue NDV. We repeated the work and obtained similar results.

In this recovery manipulation, the first strand of viral genome-like RNA was transcribed from the full-length clone by T7 RNA polymerase in the cytoplasm of BSR T7/5. Then, the HDV ribozyme located in the 3' end of the transcribed RNA cut itself out together with the unrequired RNA fragment. In this way, a negative strand RNA with no methylation at its 5 ' end and no polyadenylation at its 3' end was generated, resembling the genomic (-) RNA of NDV. Meanwhile, vRdRp of 9a5b was assembled from the NP, P and L proteins encoded by co-transfected helper plasmids. Using (-) viral RNA as a template, the vRdRp generated $(+)$ viral RNA, together with all eight strands of viral mRNAs, which encoded the viral proteins required for the generation of infectious viral particles.

\section{Conclusion}

In this study, we constructed a full-length cDNA clone and the helper plasmids pCI-NP, pCI-P, and pCI-L from cDNA of 9a5b-D5C1. After these plasmids were co-transfected into BSR T7/5 cells, infectious viral particles were obtained. The rescued viruses were genetically and biologically identical to the parental strain and showed similar pathogenicity in chickens, suggesting that a stable recovery manipulation for class I NDV had been established. The reverse genetics manipulation of $9 \mathrm{a} 5 \mathrm{~b}$ developed here allows the generation of genetically altered NDV, which can be used for research on the evolution of class I NDV isolates.

\section{Methods}

\section{Virus, plasmids and cells}

The virulent NDV strain 9a5b was obtained from Professors Toshihiro Ito and Koichi Otsuki in Tottori University, Japan [31]. The virus was plaque-purified five times in DF1 cells, and the final pure clone used in this study was named D5C1 and was grown for 9-11 days in SPF embryonated eggs. BSR T7/5 cells were obtained from Dr. Zhigao $\mathrm{Bu}$ in Harbin Veterinary Research Institute, Chinese Academy of Agricultural Sciences, China. The cells were developed by Buchholz et al [33] and they express the phage T7 RNA polymerase in a stable manner in culture. The cells were maintained in DMEM (Gibco, USA) supplemented with $10 \%$ fetal calf serum (FCS) and $1 \mathrm{mg} / \mathrm{ml} \mathrm{G418} \mathrm{(Sigma,} \mathrm{Saint} \mathrm{Louis,} \mathrm{MO,}$ USA), as previously reported [20,33,34]. The vector pTVT7R $(0,0)$ was a generous gift from Andrew Ball $[20,34]$. In this vector, the T7 promoter sequence and the hepatitis delta virus (HDV) ribozyme sequence flank the insertion site for the full-length NDV cDNA (Figure 1A). After it was transcribed from the plasmid with T7 polymerase in the BSR T7/5 cells, the resulting RNA is an exact copy of the parental NDV genome $[34,35]$.

\section{Cloning and assembly of the full-length NDV cDNA clone}

Fresh allantoic fluid from the D5C1 virus was directly utilized for the isolation of viral genomic RNA using the Trizol RNA extraction kit (Invitrogen, Carlsbad, CA, USA). The cDNA was reverse transcribed from viral RNA with a 6-nt random primer or a specific primer (5'-ACC AAA CAG AGA ATC-3') complementary to the 3 ' end of the NDV genomic RNA.

In order to determine the exact full-length genomic sequence of the virus, nine successive and overlapping DNA fragments were amplified by PCR with a set of specific primer pairs for class I NDV. The 3 ' and 5 ' ends 
of the viral genome were obtained by T4 RNA ligasemediated rapid amplification of the cDNA end (RLMRACE). All of the above experiments were performed as reported previously [26]. RT-PCR products from overlapping fragments covering the full-length genome were extracted from the agarose gel, ligated into the TA cloning system (Promega, Madison, WI, USA) and transferred into E. coli DH5 $\alpha$. At least five clones of each segment were sent to Sangon Biotechnology (Shanghai, China) for sequencing. The sequences of overlapping DNA fragments were aligned and then compiled into a correct and precise consensus full-length genome sequence using the Lasergene software package (DNASTAR Inc. Madison, WI 53715, USA).

According to the restriction enzyme site maps, a set of primers was designed to amplify nine overlapping fragments (Table 1). Segment Le and Tr, flanked by the BsmBI and SpeI restriction enzyme sites respectively, were digested and ligated with BbsI-digested vector pTVT7R(0.0) to construct plasmid TVT-3'5'. Segment A, which was the ligation product of segments A1, A2 and A3, was digested by AgeI and StuI and then ligated into TVT-3'5' to obtain plasmid TVT-A. Segments B1, B2 and B3 were also inserted into TVT-3'5' in order to obtain TVT-B. Finally, a full-length cDNA clone, TVTD5C1, was completed via ligation of AgeI-Sall doubledigested TVT-A and TVT-B (Figure 1C).

\section{Construction of helper plasmids}

The full-length cDNA clone was utilized as a template to amplify the NP, P, and L genes, which were cloned into the eukaryotic expression vector pCI neo (Promega, Madison, WI, USA) to generate three helper plasmids.

To clone the NP and P genes, two primer pairs of PNF (5'-GA CTC GAG ATG TCC TCC GTA TTC GA-3') and PNR (5'- T TCT AGA CTA GTA TCC CCA GTC GG -3'), and PPF (5'-TA CTC GAG AGC ATG GCT ACG TTC AC-3') and PPR (5'-G $A C G$ CGT TTA TCC ATT CAG TGC AA-3') (XhoI, XbaI and $M l u$ I sites are in italics and underlined) were used for PCR with AccuPrime $^{\text {Tx }}$ Taq DNA Polymerase High Fidelity (Invitrogen); the PCR products were double-digested and then inserted in the multiple-cloning site of pCI-neo (Promega, Madison, WI, USA).

To clone the entire L gene, three overlapping segments, L1, L2 and L3, were amplified with three primer pairs: PL1F (5'-CGC GCT AGC AGA CCA TGG CAG GCT CCG GGC CC-3') and PL1R (5'-GTG GTT GAT GGC ATG CAT GAG CGA ATA G-3'), PL2F (5'-AAT GAC AAC CCG CGG CGT GAA CTG CTA TC-3') and PL2R (5'-CAG CAC AAC ATG GCA GCG GA-3'), and PL3F (5'-GCA CGA GCT AGA CTT AAG CGC TCC AGC ACG-3') and PL3R (5'-TAA GGT CGA CAA TCA ACA GCA TTT ACC ACT A-3'). The segment L2 was digested and cloned into the vector pCI-neo between the MluI and NheI sites. NheI-digested segment L2 and double-digested segment L3 were subsequently ligated into the vector in order.

\section{Construction of a minigenome plasmid for NDV D5C1}

NDV D5C1 with a reporter gene of GPF was constructed. Three primer pairs, described below, were designed to amplify three sub-fragments.

Table 1 Primers used in this study to generate overlapping PCR fragments from the genome of D5C1

\begin{tabular}{|c|c|c|c|c|}
\hline Fragment designation & & Primer sequence $\left(5^{\prime}-3^{\prime}\right)$ & Position $^{c}$ & $\begin{array}{l}\text { Expected product } \\
\text { size (bp) }\end{array}$ \\
\hline \multirow[t]{2}{*}{ Le } & $\mathrm{F}^{\mathrm{a}}$ & ACCAAACAGAGAATCCGTGAG & $1-21$ & 2280 \\
\hline & $R^{b}$ & TGGACGATTTATTGCTAAGCTTG & $2258-2280$ & \\
\hline \multirow[t]{2}{*}{ B1 } & $\mathrm{F}$ & CAAGACTGGAGCAAGCAACT & $2219-2238$ & 2003 \\
\hline & $\mathrm{R}$ & GGAGAGGCATTTGCTATAGG & $4202-4221$ & \\
\hline \multirow[t]{2}{*}{ B2 } & $\mathrm{F}$ & GGGCTCAGTGATGTGCTCG & $4100-4118$ & 1964 \\
\hline & $\mathrm{R}$ & ATATAGGTAATGAGAGCAGATGTG & $6040-6063$ & \\
\hline \multirow[t]{2}{*}{$\mathrm{B} 3$} & $\mathrm{~F}$ & AAATAATATGCGTGCCACCT & $5434-5453$ & 1661 \\
\hline & $\mathrm{R}$ & GAACGCAGAGTAGAAAAGAATA & 7073-7094 & \\
\hline \multirow[t]{2}{*}{$\overline{A 1}$} & $\mathrm{~F}$ & CAAGAACACCTGAATTTTATCCCG & 6686-6909 & 2260 \\
\hline & $\mathrm{R}$ & TTAGATGCCTTTGGACCTGTTTA & $8922-8945$ & \\
\hline \multirow[t]{2}{*}{ A2 } & $\mathrm{F}$ & TGGTITCACTCAAAATGGTCC & $8876-8896$ & 1223 \\
\hline & $\mathrm{R}$ & ATCCCTTCTGCCATTACCTG & 10079-10098 & \\
\hline \multirow[t]{2}{*}{$\overline{A 3}$} & $\mathrm{~F}$ & ACCCTTGAGTACCTAAGAGATGA & $9965-9987$ & 2116 \\
\hline & $\mathrm{R}$ & TGTCCCCATAAGCCCAGAT & $12062-12080$ & \\
\hline \multirow[t]{2}{*}{$\mathrm{Tr}$} & $\mathrm{F}$ & CTAGGAAGAGCCTTAATTTGAT & 13350-13371 & 1838 \\
\hline & $R$ & ACAAAGATTTGGTGAATGACA & $15167-15187$ & \\
\hline
\end{tabular}

\footnotetext{
${ }^{\mathrm{a}} \mathrm{F}$ represents forward primer; ${ }^{\mathrm{b}} \mathrm{R}$ represents reverse primer; ${ }^{\mathrm{c}}$ Positions were based on the genomic sequence of NDV D5C1 (accession number: JQ713944).
} 
The first pair, primers MLF (5'-CGT CTC TAC CCA CCA AAC AGA GAA TCT GTA AGG TAC GAT ATA AG-3') and MLR (5'-ACC ATC CCG GGA CGC GTA GAA GGT ACT CTC GAG CTT GAG CTT C-3'), were used to amplify fragment MLe from plasmid TVT-3'5' constructed above. The PCR product of MLe contained the sequence of leader and 5' UTR of NP protein, located from $1 \mathrm{nt}$ to $121 \mathrm{nt}$ of the viral genome of D5C1. The BsmBI site (underlined) was added in the 5 ' end of primer MLF.

The second pair, primers MGF (5'-TTC TAC GCG TCC CGG GAT GGT GAG CAA GGG CGA GGA GC TGT T-3') and MGR (5'-GTT ACA GGT ACC GAT ATC TTA CTT GTA CAG CTC GTC CAT GCC G-3'), were used to amplify the fragment GFP from plasmid pEGFP-N1 vector (CLONTECH Laboratories, Inc.).

The third pair, primers MTF (5'-AAG TAA GAT ATC GGT ACC TGT AAC TGT TGA TAA TAG TGG TAA ATG C-3') and primer MTR (5'-T CGT CTC GTA TAG GGA CCA AAC AAA GAT TTG GTG AAT ATC AG-3'), were used to amplify fragment MTr from plasmid TVT-3'5' constructed above. The PCR product of MTr contained the sequence of 3' UTR of L protein and leader, located from $15,008 \mathrm{nt}$ to $15,198 \mathrm{nt}$ in the viral genome of $\mathrm{D} 5 \mathrm{C} 1$. The $\mathrm{BsmBI}$ site (underlined) and three extra $\mathrm{G}$ residues (bold) were added in the 5 ' end of primer MTR.

All three sub-fragments were joined together with primers MTF and MTR by overlapping PCR. The fusion fragment was digested with $B s m B I$ and then ligated with BbsI-digested pTVT7R $(0.0)$ vector. The recombinant plasmid was sent to Sangon Biotechnology (Shanghai, China) for sequencing.

\section{Cotransfection of helper plasmids with TVT-D5C1 or TVT-TGL}

High-quality plasmid DNA from TVT-D5C1 and three helper plasmids were obtained from purification using QIAGEN Plasmid Midi Kits. BSR T7/5 cells were grown to $60 \%$ confluency in $35 \mathrm{~mm}$ diameter dishes and then transfected with a total of $3 \mu \mathrm{g}$ of plasmids using Lipofectamine $^{\mathrm{TM}} 2000$ Reagent (Invitrogen) according to the manufacturer's instructions. To determine the activity of the helper plasmid, $1 \mu \mathrm{g}$ of pCI-NP, $0.5 \mu \mathrm{g}$ of pCI-P and $0.5 \mu \mathrm{g}$ of $\mathrm{pCI}-\mathrm{L}$ were mixed with $1 \mu \mathrm{g}$ of minigenome plasmid TVT-TGL in the transfection. Then, in the rescue process, $1 \mu \mathrm{g}$ of TVT-D5C1 was utilized instead of pTVT-TGL to generate the recombinant virus. The cells were observed every $12 \mathrm{~h}$ for the expression of fluorescence and harvested at $72 \mathrm{~h}$ post-transfection for propagation in eggs. These materials were then inoculated into the allantoic cavity of 10-day-old embryonated SPF chicken eggs to enable the efficient propagation of recovered recombinant viruses. Three successive passages in embryonated SFP chicken eggs were performed with a volume of $100 \mu \mathrm{l}$ for each egg. All of the allantoic fluid from these eggs was harvested for further tests. The allantoic fluid harvested from each inoculated egg was tested for hemagglutination (HA) and hemagglutinin inhibition (HI) titers; for these tests, anti-NDV serum collected from NDV-immunized SPF chickens was used.

\section{Characterization of the rescued viruses}

Viral RNA from the rescued viruses was extracted from fresh allantoic fluid following RT-PCR, and the fulllength sequencing was performed as described above. The full-length genomic sequence was obtained from nine overlapping fragments. The viruses propagating in these eggs were genetically identified by comparison with the sequences of the parental virus.

Mean death time (MDT), intracerebral pathogenicity index (ICPI) and intravenous pathogenicity index (IVPI) were calculated to determine the virulence of rescued viruses, as described by the world organization for animal health (OIE) [36]. In each test, the parental virus $9 \mathrm{a} 5 \mathrm{~b}$ was utilized as the positive control. To determine the MDT value, the virus was serial 10 -fold diluted, and then each dilution was inoculated into the allantoic cavities of five 9- to 11-day-old SFP embryonated eggs. Those inoculated eggs were incubated at $37{ }^{\circ} \mathrm{C}$ and checked every $12 \mathrm{~h}$ for 7 days. The MDT was the mean time in hours for the embryo death in the highest dilution at which all eggs died.

To determine the ICPI value, $0.05 \mathrm{ml}$ of serial diluted virus was injected intracerebrally into each of ten oneday old SPF chickens. The birds were scored for disease symptoms and mortality ( 0 for normal, 1 for sick, and 2 for dead) every $24 \mathrm{~h}$ for 8 days. The ICPI is the mean score per bird per observation over the 8-day period.

To determine the IVPI value, viruses were inoculated intravenously into ten 6-week old SPF chickens. The birds are scored for disease symptoms and mortality ( 0 if normal, 1 if sick, 2 if paralyzed, and 3 if dead) over a period of 10 days. The IVPI value is the mean score per bird per observation.

Care and maintenance of all chickens were in accordance with the animal study protocol 11-08 of the Institutional Animal Care and Use Committee (IACUS) guidelines set by Shanghai Veterinary Research Institute, the Chinese Academy of Agricultural Sciences (CAAS).

\section{Growth kinetics of recovered virus and its parental virus}

The propagation efficiency of recovered NDV and the parental virus were compared via a multiple-step growth cycle in DF-1 cells. DF-1 cells at $80 \%$ confluency were transfected with each virus at a multiplicity of infection (MOI) of 0.01 , and the supernatant was harvested at 2, $4,6,8,12,16,24,36$, and 48 h post-infection. A $50 \%$ 
tissue culture infection dose $\left(\mathrm{TCID}_{50}\right)$ test was performed to quantify the live viruses secreted into the supernatant. Briefly, virus was serially diluted 10 -fold and inoculated on DF-1 cells in 96-well plates. At 3 days post-infection, the lesions in the cells were observed under a microscope. Viral $\mathrm{TCID}_{50}$ titer in cell culture was calculated by the Reed and Muench method [37]. The experiment was performed in triplicate and a kinetic curve based on respective $\mathrm{TCID}_{50}$ was constructed for each strain.

\author{
Abbreviations \\ APMV: avian paramyxovirus; CEFs: chicken embryo fibroblasts; F: fusion \\ protein; GFP: green fluorescence protein; HA: hemagglutination; \\ $\mathrm{HI}$ : hemagglutinin inhibition; HN: hemagglutinin-neuraminidase; \\ ICPI: intracerebral pathogenicity index; L: large protein; M: matrix protein; \\ MDT: mean death time; MOl: multiplicity of infection; NCR: non-coding \\ region; ND: Newcastle disease; NDV: Newcastle disease virus; \\ NP: nucleoprotein; OIE: the world organization for animal health; \\ P: phosphoprotein; SPF: specific-pathogen-free; RACE: rapid amplification of \\ CDNA ends; RT-PCR: reverse-transcription polymerase chain reaction; \\ SPF: specific-pathogen-free; $T_{C I D}$ : $50 \%$ tissue culture infection dose; \\ UTR: untranslated region.
}

\section{Competing interests}

The authors declare that they have no competing interests.

\section{Authors' contributions}

YY contributed for the construction of all the plasmids. XQ coordinated with the experiment design and drafted the manuscript. DX and $\mathrm{HC}$ carried out the construction of full-length NDV CDNA clone. YZ, LT and CM constructed the helper plasmids. NW performed the virus propagation in embryonated eggs. SY contributed manuscript editing. CD, XL and AQ made substantial contributions to the experiment design. All authors' have read and approved the final manuscript.

\section{Acknowledgements}

We would like to thank Professors Toshihiro Ito and Koichi Otsuki from Tottori University, Japan, for providing us with NDV strains. And also, we would like to thank Dr. Zhigao Bu, from Harbin Veterinary Institute, China and Andrew Ball, from Department of Microbiology, University of Alabama at Birmingham, Birmingham, Alabama, for providing us with BSR T7/5 and $\operatorname{TVT7R}(0,0)$. This work was funded by Chinese Special Fund for Agroscientific Research in the Public Interest (201003012) and Chinese National High-tech R\&D Program (863 Program, 2011AA10A209).

\section{Author details}

${ }^{1}$ College of Veterinary Medicine, Yangzhou University, 48 Wenhuidong Road, Yangzhou 225009, People's Republic of China. ${ }^{2}$ Shanghai Veterinary Research Institute, Chinese Academy of Agricultural Sciences, Shanghai 200241, People's Republic of China. ${ }^{3}$ China National Engineering Technology Research Centre for Poultry, 2949 Zhennan Road, Shanghai 200331, People's Republic of China.

Received: 24 October 2011 Accepted: 11 June 2012

Published: 18 June 2012

\section{References}

1. Alexander DJ: Newcastle disease, other avian paramyxoviruses, and pneumovirus infections. In Diseases of Poultry. 11th edition. Edited by Saif JM, Barnes HJ, Glisson JR, Fadly AM, McDougald LR, Swayne DE. Ames, lowa: lowa State University Press; 2003:63-69.

2. Shimazu Y, Takao SI, Irie T, Kiyotani K, Yoshida T, Sakaguchi T: Contribution of the leader sequence to homologous viral interference among Sendai virus strains. Virology 2008, 372:64-71.

3. Aldous EW, Alexander DJ: Detection and differentiation of Newcastle disease virus (avian paramyxovirus type 1). Avian Pathol 2001, 30:117-128.
4. de Leeuw O, Peeters B: Complete nucleotide sequence of Newcastle disease virus: evidence for the existence of a new genus within the subfamily Paramyxovirinae. J Gen Virol 1999, 80(Pt 1):131-136.

5. Mayo MA: A summary of taxonomic changes recently approved by ICTV. Arch Virol 2002, 147:1655-1663.

6. Millar NS, Chambers P, Emmerson PT: Nucleotide sequence of the fusion and haemagglutinin-neuraminidase glycoprotein genes of Newcastle disease virus, strain Ulster: molecular basis for variations in pathogenicity between strains. J Gen Virol 1988, 69(Pt 3):613-620.

7. Samson AC, Levesley I, Russell PH: The $36 \mathrm{~K}$ polypeptide synthesized in Newcastle disease virus-infected cells possesses properties predicted for the hypothesized 'V' protein. J Gen Virol 1991, 72(Pt 7):1709-1713.

8. Steward M, Vipond IB, Millar NS, Emmerson PT: RNA editing in Newcastle disease virus. J Gen Virol 1993, 74(Pt 12):2539-2547.

9. Gould AR, Hansson E, Selleck K, Kattenbelt JA, Mackenzie M, Della-Porta AJ: Newcastle disease virus fusion and haemagglutinin-neuraminidase gene motifs as markers for viral lineage. Avian Pathol 2003, 32:361-373.

10. Czegledi A, Ujvari D, Somogyi E, Wehmann E, Werner O, Lomniczi B: Third genome size category of avian paramyxovirus serotype 1 (Newcastle disease virus) and evolutionary implications. Virus Res 2006, 120:36-48.

11. Miller PJ, Decanini EL, Afonso CL: Newcastle disease: evolution of genotypes and the related diagnostic challenges. Infect Genet Evol, 10:26-35.

12. Mia Kim L, King DJ, Suarez DL, Wong CW, Afonso CL: Characterization of class I Newcastle disease virus isolates from Hong Kong live bird markets and detection using real-time reverse transcription-PCR. J Clin Microbiol 2007, 45:1310-1314.

13. Liu H, Chen F, Zhao Y, Zheng D, Li J, Xu T, Qi L, Wang Z: Genomic characterization of the first class I Newcastle disease virus isolated from the mainland of China. Virus Genes 2010, 40:365-371.

14. Devine MJ, Kaganovich A, Ryten M, Mamais A, Trabzuni D, Manzoni C, McGoldrick P, Chan D, Dillman A, Zerle J, et al: Pathogenic LRRK2 mutations do not alter gene expression in cell model systems or human brain tissue. PloS One 2011, 6:e22489.

15. Rotheray KR, Cheung PS, Cheung CS, Wai AK, Chan DY, Rainer TH, Graham CA: What is the relationship between the Glasgow coma scale and airway protective reflexes in the Chinese population? Resuscitation 2011.

16. Lin CT, Hsu KF, Jao SW, Chen TW, Yu JC, Hsieh CB, Chan DC: Leukemoid reaction with metachronous tumors. Rev Esp Enferm Dig 2011, 103:384-385.

17. Li BY, Li XR, Lan X, Yin XP, Li ZY, Yang B, Liu JX: Rescue of Newcastle disease virus from cloned cDNA using an RNA polymerase II promoter. Arch Virol 2011, 156:979-986.

18. Peeters BP, de Leeuw OS, Koch G, Gielkens AL: Rescue of Newcastle disease virus from cloned CDNA: evidence that cleavability of the fusion protein is a major determinant for virulence. J Virol 1999, 73:5001-5009.

19. Nakaya T, Cros J, Park MS, Nakaya Y, Zheng H, Sagrera A, Villar E, GarciaSastre A, Palese P: Recombinant Newcastle disease virus as a vaccine vector. J Virol 2001, 75:11868-11873.

20. Liu YL, Hu SL, Zhang YM, Sun SJ, Romer-Oberdorfer A, Veits J, Wu YT, Wan HQ, Liu XF: Generation of a velogenic Newcastle disease virus from CDNA and expression of the green fluorescent protein. Arch Virol 2007, 152:1241-1249.

21. de Leeuw OS, Koch G, Hartog L, Ravenshorst N, Peeters BP: Virulence of Newcastle disease virus is determined by the cleavage site of the fusion protein and by both the stem region and globular head of the haemagglutinin-neuraminidase protein. J Gen Virol 2005, 86:1759-1769.

22. Romer-Oberdorfer A, Mundt E, Mebatsion T, Buchholz UJ, Mettenleiter TC: Generation of recombinant lentogenic Newcastle disease virus from cDNA. J Gen Virol 1999, 80(Pt 11):2987-2995.

23. Mattarollo SR, Yong M, Gosmann C, Choyce A, Chan D, Leggatt GR, Frazer IH: NKT cells inhibit antigen-specific effector CD8 T cell induction to skin viral proteins. J Immunol 2011, 187:1601-1608.

24. Chan D, Tarbin J, Sharman M, Carson M, Smith M, Smith S: Screening method for the analysis of antiviral drugs in poultry tissues using zwitterionic hydrophilic interaction liquid chromatography/tandem mass spectrometry. Anal Chim Acta 2011, 700:194-200.

25. Huang Y, Wan HQ, Liu HQ, Wu YT, Liu XF: Genomic sequence of an isolate of Newcastle disease virus isolated from an outbreak in geese: a novel 
six nucleotide insertion in the non-coding region of the nucleoprotein gene. Brief Report. Arch Virol 2004, 149:1445-1457.

26. Qiu X, Sun Q, Wu S, Dong L, Hu S, Meng C, Wu Y, Liu X: Entire genome sequence analysis of genotype IX Newcastle disease viruses reveals their early-genotype phylogenetic position and recent-genotype genome size. Virol J 2011, 8:117.

27. Vakarelski IU, Marston JO, Chan DY, Thoroddsen ST: Drag reduction by Leidenfrost vapor layers. Phys Rev Lett 2011, 106:214501.

28. Zhang H, Chan DK, Teng F: Transfer of attachment functions and adjustment among young adults in China. J Soc Psychol 2011, 151:257-273.

29. Liu X, Wang X, Wu S, Hu S, Peng Y, Xue F: Surveillance for avirulent Newcastle disease viruses in domestic ducks (Anas platyrhynchos and Cairina moschata) at live bird markets in Eastern China and characterization of the viruses isolated. Avian Pathol 2009, 38:377-391.

30. Alexander DJ, Campbell G, Manvell RJ, Collins MS, Parsons G, McNulty MS: Characterisation of an antigenically unusual virus responsible for two outbreaks of Newcastle disease in the Republic of Ireland in 1990. Vet Rec 1992, 130:65-68.

31. Shengqing $\mathrm{Y}$, Kishida $\mathrm{N}$, Ito H, Kida H, Otsuki K, Kawaoka Y, Ito T: Generation of velogenic Newcastle disease viruses from a nonpathogenic waterfowl isolate by passaging in chickens. Virology 2002, 301:206-211.

32. Tsunekuni R, Ito H, Otsuki K, Kida H, Ito T: Genetic comparisons between lentogenic Newcastle disease virus isolated from waterfowl and velogenic variants. Virus Genes 2010, 40:252-255.

33. Buchholz UJ, Finke S, Conzelmann KK: Generation of bovine respiratory syncytial virus (BRSV) from cDNA: BRSV NS2 is not essential for virus replication in tissue culture, and the human RSV leader region acts as a functional BRSV genome promoter. J Virol 1999, 73:251-259.

34. Johnson KN, Zeddam JL, Ball LA: Characterization and construction of functional cDNA clones of Pariacoto virus, the first Alphanodavirus isolated outside Australasia. J Virol 2000, 74:5123-5132.

35. Krishnamurthy S, Huang Z, Samal SK: Recovery of a virulent strain of newcastle disease virus from cloned CDNA: expression of a foreign gene results in growth retardation and attenuation. Virology 2000, 278:168-182.

36. Lacorte JG: Ophthalmotropic viruses; trachoma virus and inclusion conjunctivitis virus. Rev Bras Med 1951, 8:334-341.

37. Reed MJ, Muench $\mathrm{H}$ : A simple method of estimating fifty percent endpoints. Am J Hyg 1938, 27:493-397.

doi:10.1186/1743-422X-9-120

Cite this article as: Yu et al.: Rescue of virulent class I Newcastle disease

virus variant 9a5b-D5C1. Virology Journal 2012 9:120.

\section{Submit your next manuscript to BioMed Central and take full advantage of:}

- Convenient online submission

- Thorough peer review

- No space constraints or color figure charges

- Immediate publication on acceptance

- Inclusion in PubMed, CAS, Scopus and Google Scholar

- Research which is freely available for redistribution 